OPEN ACCESS

Edited by:

Timothy F. Burns,

University of Pittsburgh, United States

Reviewed by:

Anthony Faber,

Virginia Commonwealth University,

United States

Yuichi lida,

Shimane University, Japan

*Correspondence:

Haihua Yang

yhh93181@hotmail.com

Specialty section:

This article was submitted to

Cancer Molecular

Targets and Therapeutics,

a section of the journal

Frontiers in Oncology

Received: 06 February 2021 Accepted: 20 April 2021

Published: 08 June 2021

Citation:

Wang W, Xia X, Chen K, Chen M, Meng Y, Lv D and Yang H (2021) Reduced PHLPP Expression Leads to

EGFR-TKI Resistance in Lung Cancer by Activating PI3K-AKT and MAPK-ERK Dual Signaling.

Front. Oncol. 11:665045. doi: 10.3389/fonc.2021.665045

\section{Reduced PHLPP Expression Leads to EGFR-TKI Resistance in Lung Cancer by Activating PI3K-AKT and MAPK- ERK Dual Signaling}

\author{
Wei Wang ${ }^{1,2}$, Xinhang Xia ${ }^{1,2}$, Kuifei Chen ${ }^{3}$, Meng $C{ }^{3}{ }^{3}$, Yinnan Meng ${ }^{1,2}$, Dongqing $L^{4}{ }^{4}$ \\ and Haihua Yang ${ }^{1,2,3 *}$ \\ ${ }^{1}$ Laboratory of Cellular and Molecular Radiation Oncology, Radiation Oncology Institute of Enze Medical Health Academy, \\ Affiliated Taizhou Hospital of Wenzhou Medical University, Taizhou, China, ${ }^{2}$ Department of Radiation Oncology, Affiliated \\ Taizhou Hospital of Wenzhou Medical University, Taizhou, China, ${ }^{3}$ School of Medicine, Shaoxing University, Shaoxing. China, \\ ${ }^{4}$ Department of Pulmonary Medicine, at Enze Hospital, Affiliated Taizhou Hospital of Wenzhou Medical University, \\ Taizhou, China
}

Background: Epidermal growth factor receptor tyrosine kinase inhibitors (EGFR-TKIs) are effective in advanced EGFR-mutation non-small cell lung cancer (NSCLC) but the magnitude of tumor regression varies, and drug resistance is unavoidable. The pleckstrin homology domain leucine-rich repeat protein phosphatase (PHLPP) levels are reduced or lost and acts as a tumor suppressor in many cancers. Here, we hypothesized that PHLPP is a key regulator of EGFR-TKI sensitivity and a potential treatment target for overcoming resistance to EGFR-TKI in lung cancer.

Methods: Cell proliferation and growth inhibition were measured by 3-(4,5dimethylthiazol-2-yl)-2,5-diphenyltetrazolium bromide (MTT) and colony formation assay. PHLPP- knockdown stable cell lines were generated by lentivirus-mediated delivery of PHLPP shRNAs. The expression of PHLPP mRNA and protein levels was detected by real-time quantitative polymerase chain reaction (qPCR) and Western blotting. Immunohistochemical $(\mathrm{IHC})$ staining was performed to detect the PHLPP expression in clinical patient tissue samples. A transcriptomic assay of genome-wide RNA expressions of PHLPP in NSCLC cell lines according to gefitinib sensitivity was obtained from Gene Expression Omnibus (GEO) database. Murine xenograft model was established to verify the function of PHLPP in gefitinib resistance in vivo.

Results: PHLPP highly expressed in gefitinib-sensitive NSCLC cell lines than gefitinibresistant NSCLC cell lines. In gefitinib-acquired resistance cell line HCC827-GR, PHLPP expression even dramatically reduced. Knockdown of PHLPP in NSCLC cells decreased cell death induced by the EGFR-TKI, while overexpression PHLPP in gefitinib-resistance NSCLC cells can enhance or restore EGFR-TKls sensitivity. Mechanism study indicated that PHLPP downregulation attenuates the effect of EGFR-TKI on the both AKT and ERK pathway, thereby decreasing the cell death sensitivity to EGFR inhibitors. In xenograft mice, knockdown of PHLPP decreased tumor response to gefitinib and advanced tumor 
cells re-growth after gefitinib treatment. In clinical, PHLPP expression were reduced in the post-relapse tumor compared to that of pre-treatment, and lower pre-treatment PHLPP levels were significantly correlated with shorter progression-free survival (PFS) in patients with EGFR-mutant lung adenocarcinoma whom treated with EGFR-TKI.

Conclusions: Our data strongly demonstrated that loss of PHLPP function was a key factor of EGFR-TKI resistance in NSCLC. Downregulated PHLPP expression activated PI3K-AKT and MAPK-ERK pathway which strengthened cell survival to EGFR-TKI. Therefore, PHLPP expression level was not only a potential biomarker to predict EGFR-TKIs sensitivity but also as a therapeutic target in EGFR-TKIs therapy, enhancing PHLPP expression may be a valuable strategy for delaying or overcoming EGFR-TKIs drug resistance.

Keywords: lung cancer, PHLPP, EGFR-TKI resistance, signaling pathway, biomaker

\section{BACKGROUND}

Lung cancer is the leading cause of cancer-related death worldwide and non-small cell lung cancer (NSCLC) is the most histological subtype of lung carcinoma (1). Lung adenocarcinoma (LUAD) accounts for $60 \%$ in lung cancer. Mutations in EGFR are present in $40 \%$ to $60 \%$ of East Asianancestry LUADs (2). EGFR tyrosine kinase inhibitors (TKIs), such as erlotinib, gefitinib, afatinib, osimertinib, and so on, all were widely used in Chinese and other Asian LUAD patients (3-6). Although the achievement of this treatment is significantly effective, the patients' overall survival after treatment remains poor due to the TKI resistance. Therefore, identifying a reliable biomarker for predicting primary TKI sensitivity/resistance and method to delay acquire resistance onset is essential important.

Many mechanisms of acquired resistance have been identified over the last several decades. One of the common resistance mechanisms is the development of secondary mutation in EGFR, such as T790M mutation, which is detected in more than 50\% EGFR-TKIs resistance cases (7). The second category of resistance is associated with re-activation of the two main EGFR downstream signaling pathways PI3K-AKT and MEK/ ERK, such as MET was found to promote resistance by reactivating both $\mathrm{PI} 3 \mathrm{~K}-\mathrm{AKT}$ and MEK/ERK signaling despite the inhibition of EGFR (8); In addition, tumor resistance undergoes cell/tumor phenotypic/histological changes, such as epithelial-to-mesenchymal transition (EMT) histological transformation and development toward small-cell lung cancer (SCLC) (9-11). However, resistant mechanism in about 30\% recurrence patients is still unknown.

$\mathrm{PH}$ domain leucine rich repeat protein phosphatases (PHLPP) comprises of two genes: PHLPP and PHLPP2. Both

\footnotetext{
Abbreviations: NSCLC, non-small cell lung cancer; LUAD, lung adenocarcinoma; PHLPP, pleckstrin homology domain leucine-rich repeat protein phosphatase; EGFR, epidermal growth factor receptor; IHC, immunohistochemical; GEO, Gene Expression Omnibus; PFS, progression-free survival; TKIs, tyrosine kinase inhibitors; DFS, disease-free survival; ATCC, American Type Culture Collection; IC50, the inhibition concentration of $50 \%$ cell viability; GR, gefitinib-resistant.
}

family members belong to a novel family of Ser/Thr phosphatase, which directly dephosphorylate Akt Ser473 site and inactivate the Akt kinase, and dephosphorylate Raf1 S338 then down regulate the MAPK-ERK signaling $(12,13)$. PHLPP was downregulated in various tumors, which leads to development of carcinogenesis and cancer progression. In addition, recent studies showed that restoration of PHLPP increased the sensitivity of the colorectal cancer cells to EGFR monoclonal antibodies cetuximab and reverted the resistance (14). In chemotherapy, downregulation of PHLPP contributed to chemosensitivity, achieved cancer cells sensitive to oxaliplatin and paclitaxel resistance in colon cancer cell (15). In our own previous clinical results, we found that high PHLPP expression predicted longer duration of acquired resistance in lung adenocarcinoma patients with EGFR tyrosine kinase inhibitors (16). However, the role of PHLPP in resistance to EGFR-TKI in EGFR-mutation lung cancer remains undefined. Given the aforementioned studies demonstrating that PHLPP plays an important role in tumor progression and treatment resistance, it seems reasonable to speculate that TKI could not fully inhibit PHLPP loss-mediated AKT S473 phosphorylated to cause TKI resistance in PHLPP downregulation EGFR-mutant lung cancer cells.

Since the critical role of PHLPP function dampens both PI3KAKT and MAPK-ERK proliferation signaling pathways, several resistance mechanisms toward EGFR-TKI are through reactivation of PI3K-AKT and MAPK-ERK. We hypothesized that PHLPP might have an important role involved in the underlying mechanism, changing the EGFR-TKI sensitivity in harbored EGFR mutation NSCLC. In the present study, we will determine the role of PHLPP in EGFR TKI resistance lung cancer after EGFR-TKI therapy in vitro and in vivo.

\section{MATERIALS AND METHODS}

\section{Cell Culture and Reagents}

H1650, H1975, and HCC827 cell lines were purchased from the American Type Culture Collection (ATCC). All cell lines were 
test for mycoplasma and were negative for mycoplasma using mycoplasma Plus PCR Primer Set. All cells were cultured in a $37^{\circ} \mathrm{C}, 5 \% \mathrm{CO}_{2}$-humidified incubator using RPMI 1640 medium with 10\% fetal bovine serum (FBS; Gibco) and penicillin (100 $\mathrm{U} / \mathrm{ml}) /$ streptomycin $(100 \mu \mathrm{g} / \mathrm{ml})$ while HERK-293T were maintained in DMEM-completed medium. Gefitinib, erlotinib, afatinib, and osimertinib, purchased from Selleck Chemicals, were dissolved in dimethylsulfoxide (DMSO) to a final concentration of $10 \mathrm{mmol} / \mathrm{L}$ and stored at $-20^{\circ} \mathrm{C}$.

\section{Generation of Gefitinib-Resistant HCC827 Cells In Vitro}

To generate gefitinib-resistant cell line, HCC827 cells were generated and exposed to increasing concentrations of gefitinib, similar to previously described methods (17). Gefitinib concentrations were increased stepwise from $10 \mathrm{nM}$ to $2 \mu \mathrm{M}$ when the cells resumed growth kinetics similar to the untreated parental cells. Fresh drug was added every 72 h. Cells that are able to grow in $2-\mu \mathrm{M}$ gefitinib were obtained 6 months after initial drug treatment. To confirm the emergence of a resistance, MTT assay was performed following growth at each concentration after allowing the cells to grow in a drug-free condition for at least 3 days. Control HCC827 cells were maintained concomitantly without gefitinib, and their sensitivity to gefitinib was examined every month.

\section{Cell Cytotoxicity Assay}

Following the indicated drug treatment, cell viability was assessed by MTT assay. Cell suspensions $\left(2-5 \times 10^{3} / 100 \mu \mathrm{l} /\right.$ well $)$ were seeded into 96 -well cell culture plate overnight. The following day, various concentrations of indicated drugs were added. After incubation for $72 \mathrm{~h}$, cells were fixed with $4 \%$ formaldehyde for $20 \mathrm{~min}$, stained with $0.5 \%$ crystal violet solution containing $20 \%$ ( $\mathrm{vol} / \mathrm{vol}$ ) methanol in PBS, then the plates were washed with deionized water and dried completely. Crystal violet absorbed by the cells solubilized in $10 \%$ ( $\mathrm{vol} / \mathrm{vol}$ ) acetic acid were add into each well and the absorbed crystal violet optical density was detected at $595 \mathrm{~nm}$ by a microplate reader. Triplicate wells were tested at each drug concentration.

The 50\% inhibitory concentration (IC50) value was calculated using a non-linear regression model with a sigmoidal dose response using GraphPad Prism 8.0 for Windows (Prism GraphPad software, La Jolla, CA, USA). The IC50 value, defined as the concentration giving a $50 \%$ reduction in absorbance, was calculated from the survival curves. The data were graphically displayed using GraphPad Prism version 8 for Windows (Prism GraphPad software, La Jolla, CA, USA). The curves were fitted using a non-linear regression model with a sigmoidal dose response.

For colony formation, $5 \times 10^{4}$ cells/well were seeded in sixwell plate, exposed to indicated EGFT-TKI treatment and maintained in RPMI-1640 medium supplemented with $10 \%$ FBS for 14 days. Plates were fixed with $4 \%$ formaldehyde for $20 \mathrm{~min}$ at room temperature and stained in $0.5 \%$ crystal violet for $2 \mathrm{~h}$.

\section{Generation of Genetically Modified Cell Lines}

The lentivirus-mediated delivery of PHLPP shRNAs selection for stable PHLPP knockdown cell line was performed as described previous (15). pLKO-sh-PHLPP (sh97) and control vector were gifted from Dr. Gao Lab.

Lentiviral particles were produced by co-transfection of either pLKO-sh-PHLPP (sh97) or shControl Vector with pMD2G (Addgene \#12259) and psPAX2 (Addgene \#12260) into 293T cells using PEI $(1 \mu \mathrm{g} / \mu \mathrm{l})$, viral supernatants were harvested 48 and $72 \mathrm{~h}$ (second collection) after transfection, filtered with a $0.45-\mu \mathrm{m}$ cellulose acetate filter (Millipore Sigma, Cat\#SE1M002M00). Viral transductions were performed for 16 to $24 \mathrm{~h}$ in the presence of $8 \mu \mathrm{g} / \mathrm{ml}$ polybrene. Stable knockdown cells were selected $48 \mathrm{~h}$ after transduction with $2 \mu \mathrm{g} / \mathrm{ml}$ puromycin for 10 to 14 days and confirmed by qRT-PCR and Western blot.

For stable PHLPP overexpression cell line, the retrovirusesmediated delivery of overexpression plasmid encoding HAtagged PHLPP (pBabe-puro-HA-PHLPP $\beta$, From Dr. Gao, University of Kentucky) were transfected and selected with puromycin. Non-targeted retroviral vector was used as control (pBabe-puro-vector).

\section{Apoptosis Assay}

Either vehicle (DMSO) or EGFR-TKI treated cells were resuspended using phosphate-buffered saline (PBS) and stained with AnnexinV-FITC and propidium iodide (PI) using the BD apoptosis detection Kit (BD Biosciences, \#556547) according to manufacturer's instructions. Data were acquired using a CytoFlex S Flow Cytometer Beckman Coulter and analyzed with FlowJo software (TreeStar Inc.)

\section{Western Blotting}

The cells were collected and lysed in lysis buffer (50 mM Tris$\mathrm{HCl}, \mathrm{pH} 7.4 ; 100 \mathrm{mM} \mathrm{NaCl} ; 1 \mathrm{mM}$ EDTA; 1 mM EGTA; $1 \mathrm{mM}$ NaF; $0.1 \%$ SDS; $0.5 \%$ sodium deoxycholate; $1 \%$ Triton-X-100; $10 \%$ glycerol; protease and phosphatase inhibitor cocktails) on ice. Protein quantification was conducted with the BCA assay kit (Bio-Rad, Hercules, CA, USA). Equal amount of total protein lysates (about $25 \mu \mathrm{g}$ ) was subjected to $8 \%$ to $15 \%$ SDS-PAGE and transferred onto a polyvinylidene fluoride (PVDF) membrane. The PVDF membrane then was blocked with 5\% non-fat milk for $1 \mathrm{~h}$ followed by incubation with the specific primary antibodies at $4^{\circ} \mathrm{C}$ overnight. The primary antibodies are the following: PHLPP (1:2000, ProteinTech 22789-1-AP), PHLPP2 (1:2000, ProteinTech25244-1-AP), phospho-EGFR Y1068 (1:1000, CST\#3777), EGFR (1:1000, CST\#4627), phosphoERKp44/42(1:2000, CST\#4370), ERK1/2(1:1000, CST\#4695) phospho-AKT S473 (1:2000, CST \#4060), AKT(CST\#4685), $\beta$-Actin (CST\#8457). The membrane was then incubated with the horseradish peroxidase-labeled secondary antibody (ab205718, Abcam) for $1 \mathrm{~h}$ at room temperature. Signal was detected using the ECL detection kit (Thermo Fisher) and a FluoChem digital imaging system (Alpha Innotech, San Leandro, CA, USA). Band 
intensity was quantified by the software Image J (NIH, Bethesda, MD, USA).

\section{Quantitative Real-Time PCR (qRT-PCR)}

RNA was extracted by column purification kit (Qiagen RNeasy), and then retro-transcribed into first-strand cDNA using the High Capacity cDNA Reverse Transcription Kit following the manufacturer's protocol (Thermo Fisher Scientific, CAT\#4368814). qRT-PCR was performed using SYBR ${ }^{\mathrm{TM}}$ Select Master Mix (LiCfe Technologies, cat\# 4472908) on an ABI 7500 Fast Real-Time PCR platform (Applied Biosystems). Relative mRNA levels of each gene showed were normalized to the expression of the housekeeping gene GAPDH. Real-time PCR Primer as follows: PHLPP (Forward, CCTCATCCGCT TCTATGCAGG; Reverse, GCATCTTGCCTTTACGGAC); PHLPP2 (Forward, ATGGAGCAGACACTACCACTG; Reverse, GCAAAGGACGAGATGTAAGTCA); GAPDH (GG AGCGAGATCCCTCCAAAAT; Reverse, GGCTGTTG TCATACTTCTCATGG). Each sample was amplified in triplicate, and data were analyzed by relative quantitation using the $\Delta \Delta \mathrm{Ct}$ method and normalization to GAPDH.

\section{Bioinformatics Analysis of GSE4342 Database}

PHLPP and PHLPP2 transcription expression of 29 NSCLC cell lines in this study were obtained from the publicly available data set GSE4342 (18).

\section{Xenograft Mouse Studies}

All mouse studies were conducted in accordance with the standards of the Institutional Animal Care and Use Committee under a protocol approved by the Institutional Animal Care and Use Committee. Either $5 \times 10^{6}$ shRNAControl HCC827 cells or PHLPP shRNA knockdown HCC827 cells were inoculated into the low-left quadrant of the flank of 6- to 8 -week-old female nude mice. Mice were monitored daily general condition and body weight. Tumor sizes were measured every other day using calipers. Volume was calculated using the formula $\mathrm{L} \times \mathrm{W}^{2}$ $\times 0.52$, where $\mathrm{L}$ is the longest dimension and $\mathrm{W}$ is the perpendicular dimension. Mice were randomized to receive gefitinib or vehicle treatment once the mean volume was reached approximately 300 to $400 \mathrm{~mm}^{3}$. Gefitinib were administered at doses/schedules (equal to or below the maximum tolerated doses) previously reported to be effective in tumor growth inhibition (19). The experiment was terminated when the tumor in the either treatment group began to progress or when the tumor mean volume of vehicle control group reached $2000 \mathrm{~mm}^{3}$.

\section{Biopsies of Human NSCLC and Immunohistochemistry (IHC)}

This study was approved by the institutional research ethics board. All patients provided their written informed consent for permitting analyses of tissues. Patients with advanced EGFR mutation NSCLC who received EGFR-TKI as first-line treatment were identified from our institution, following clinical practice, radiographic progression on EGFR-TKI therapy underwent re-biopsy, availability of paired pre- and post-TKI FFPE biopsies. IHC was performed as described previously (20). Briefly, following deparaffinization and rehydration of the slides, antigen retrieval was performed using $10 \mathrm{mM}$ sodium citrate ( $\mathrm{pH}$ 6.0) for $5 \mathrm{~min}$. After endogenous peroxidase activity was blocked with $3 \%$ hydrogen peroxide, samples were incubated with PHLPP (1:100, ProteinTech, 25244-1-AP) overnight at $4^{\circ} \mathrm{C}, \mathrm{HRP}$ polymer for detection was subsequently applied onto the slides. Sections were then incubated with DAB refine for visualization and counterstained with hematoxylin. As previously defined, the IHC score of tumor were according to the intensity of staining with Fourier system (level 0-3: negative $=0$, weakest $=1$, moderate $=2$, strong = 3) [14].

\section{Statistical Analysis}

For the in vitro studies, statistical analysis was performed using Student's $t$ test (two-tailed) or one-way ANOVA. P $\leq 0.05$ was considered statistically significant. Values were expressed as mean \pm standard errors of the means (SEMs).

\section{RESULTS}

\section{PHLPP Expression Level Positively Correlated With Sensitivity to EGFR-TKI}

We determined that PHLPP expression level correlated with EGFR-TKI sensitivity in NSCLC cells. Western blotting showed that PHLPP expression level in the EGFR-TKI sensitivity cell line HCC827 was the highest among H1650, H1975, and HCC827 gefitinib-resistant (HCC827-GR) cells (Figures 1A, B), but cannot detect differences of the PHLPP2 protein levels in these cell lines. A similar result was determined about the PHLPP mRNA expression levels in these four cell lines (Figure 1C). The MTT assay was used to determine the inhibitive concentration of $50 \%$ cell viability (IC50) for gefitinib, as shown in Figures 1C, D. The HCC827 cells exhibited the lowest IC50 value for gefitinib $(0.035 \mu \mathrm{M})$ compared with the IC50 value for gefitinib in the H1650, H1975, and HCC827-GR were 1.27, 18.51, and 3.22 $\mu \mathrm{M}$, respectively.

Next, we analyzed the gene expression of PHLPP in NSCLC cell line according to gefitinib sensitivity from the database of GSE4342 (18), PHLPP mRNA expression was higher in the

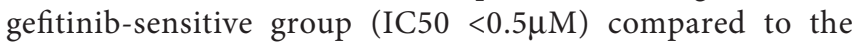
gefitinib-resistant group (IC $50 \geq 0.5 \mu \mathrm{M}$ ), the mean value of mRNA expressions were $6.5 \pm 0.35$ and $5.9 \pm 0.15$ (Figure 1E). PHLPP mRNA expression was negatively correlated with gefitinib IC50 value, cell lines with high expression of PHLPP were found to be less IC50 value of gefitinib, with a Pearson correlation score of -1.123 (Figure 1F). These findings suggested that PHLPP might be related to EGFR-TKI sensitivity in NSCLC cells.

\section{PHLPP Downregulation Re-activates ERK1/2 and AKT Signaling}

We generated a gefitinib-resistant (GR) of the EGFR-mutant HCC827 (Del E746_A750) cell line using previously established methods. Several HCC827 gefitinib-resistant (GR) clones were 
A

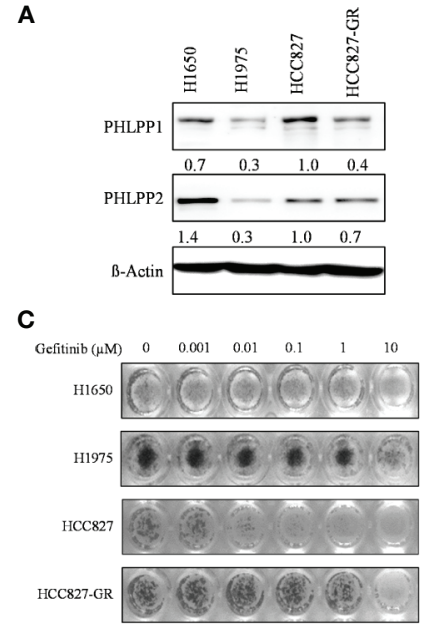

E

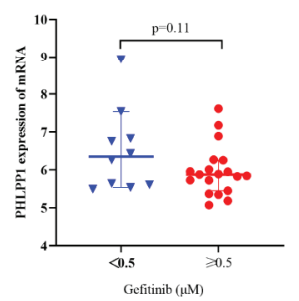

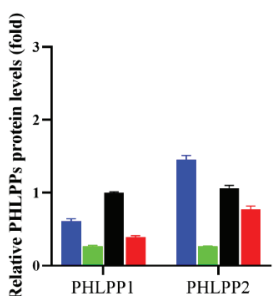

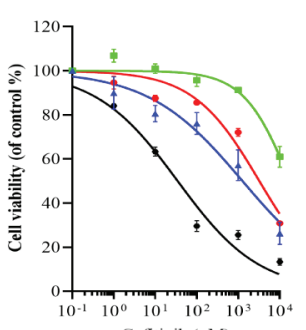

$\mathbf{F}$

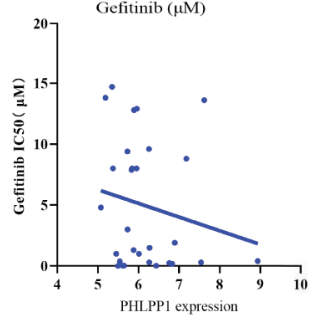

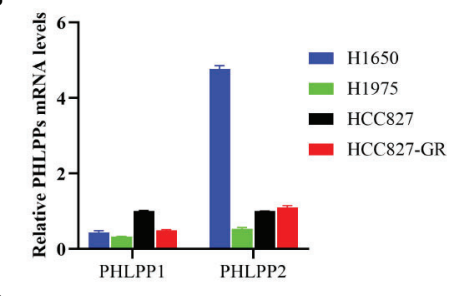

D

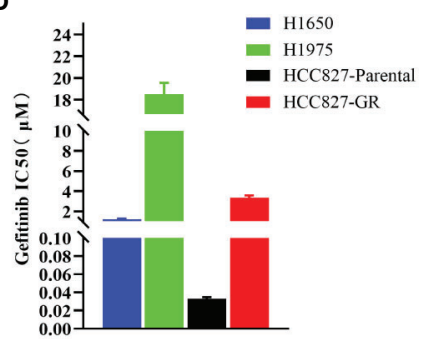

FIGURE 1 | PHLPP expression positively correlated with sensitivity to EGFR-TKI. (A) Immunoblot of PHLPP and PHLPP2 in NSCLC cell lines. $\beta$-actin was used as a loading control. The relative intensity of PHLPP levels is determined and relative to HCC827 cells. Data are from 3 independent experiments and are actin normalized and expressed relative to HCC827 cells as the mean \pm SEM. (B) Quantitative RT-PCR analysis of PHLPPs mRNA levels in NSCLC cell lines and expressed relative to HCC827 cells as the mean \pm SEM. (C) Representative image for proliferation inhibition by MTT assay(left). Five NSCLC cell lines treated with the increased concentrations of gefitinib $(0-10 \mu \mathrm{M})$, cell viability relative to vehicle treated after $72 \mathrm{~h}$. Each data point represents the mean \pm SEM of at least 3 wells(right). (D) The mean IC50 value of gefitinib from five NSCLC cell lines as measured by growth inhibition assays. Data are from three independent experiments as mean \pm SEM. (E) Box plots depicting PHLPP RNA expression in 29 NSCLC cell line form GSE4342 using the $0.5 \mu \mathrm{M}$ gefitinib IC50 values as cut-off value. (F) Dot plot depicting the correlation of gefitinib IC50 value and the expression level of PHLPP in 29 NSCLC cell lines from GSE4342.

established and confirmed to be gefitinib-resistant (Figure 2A), the IC50 of gefitinib in HCC827-GR increased 100 fold compared to HCC827-parental. The HCC827-GR cells are also cross-resistant to other EGF receptor kinase inhibitors like erlotinib, afatinib, and osimertinib (Figure 2B). All HCC827GR cells still harbored the EGFR del E746_A750 without T790M mutation. A significantly decreased expression of PHLPP in HCC827-GR cells compared with parental cells is shown in Figure 2C, but no difference was observed in PHLPP2 between these two cell types (data not shown).

To investigate the underlying mechanism how HCC827-GR is resistant to the EGFR TKI, lysates from both parental and GR cells treated with gefitinib were blotted with antibodies. We found that gefitinib still inhibited EGFR phosphorylation in the HCC827-GR cells, as well as HCC827 parental, the inhibition was decoupled from inhibition of downstream signaling both AKT and ERK phosphorylation (Figure 2D). Similar to gefitinib, another EGF receptor kinase inhibitor, erlotinib, afatinib, and osimertinib, was only able to inhibit EGFR phosphorylation but not ERK1/2 and AKT phosphorylation in the HCC827-GR cells (Figure 2E). These findings suggest that gefitinib-related EGFR TKI resistance in
HCC827 cells adopted a new mechanism by reactivating PI3KAKT and MAPK/ERK signaling, which may cause adaptive downregulation of PHLPP with long-term exposure to gefitinib.

\section{PHLPP Loss Leads to Increased EGFR-TKI Resistance In Vitro}

Given that PHLPP is downregulated in gefitinib-resistant cell line, we next to validated the possible role of PHLPP in gefitinib resistance. Stable PHLPP knockdown HCC827 cells were generated using lentiviral-based shRNA. PHLPP rival particle transductions did efficiently suppress PHLPP protein and mRNA expression levels (Figure 3A). MTT assay was used to determine the inhibition of cell proliferation in the short term induced by gefitinib in these PHLPP knockdown cells. The IC50 value was 43fold, which increased after gefitinib treatment. To test longer-term gefitinib effects in PHLPP silent HCC827 cells, we performed colony formation assays, in which PHLPP knockdown cells were culture in the presence of gefitinib for 14 days. PHLPP knockdown substantially enhanced survival in the presence of gefitinib compared to PHLPP shRNA control cell lines (Figure 3B). In addition, we found gefitinib-induced apoptosis was significantly decreased in PHLPP knockdown cells (Figure 3D). Another EGFR- 
A

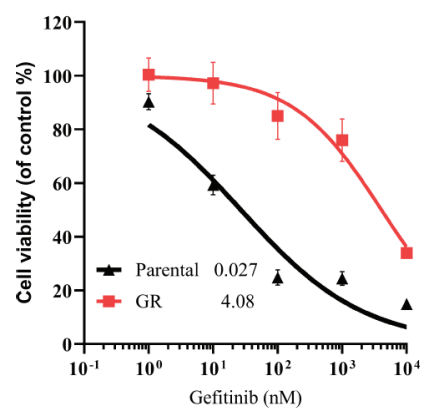

D

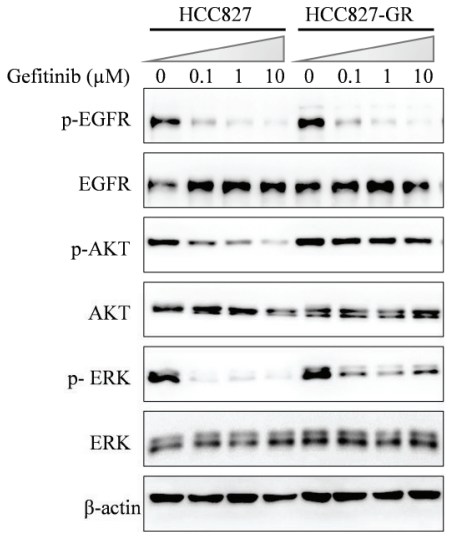

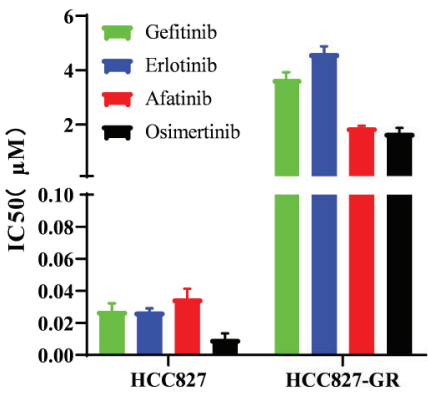

E

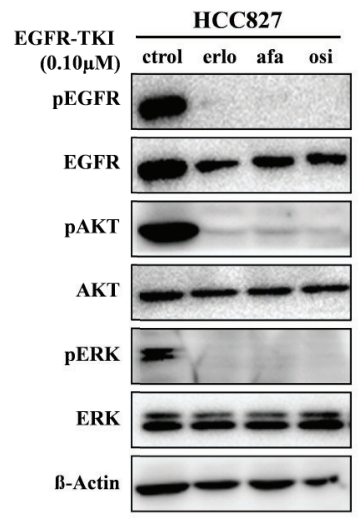

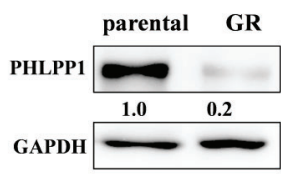

HCC827-GR
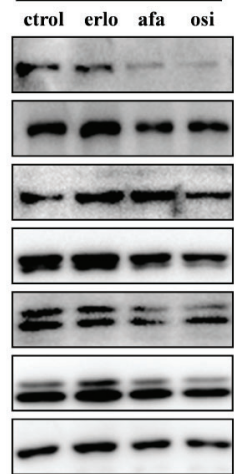

FIGURE 2 | Acquired loss of PHLPP expression in EGFR mutation cell line resistant to gefitinib. (A) Cell growth-inhibition assays demonstrate the resistance of HCC827 to gefitinib (HCC827-GR), relative to parental control HCC827. Parental HCC827 and HCC827-GR cells were subjected to a 72-h growth inhibition assay in increasing concentrations of gefitinib. Results are presented as percentage of survival compared with cell grown in the vehicle treated cells. (B) HCC827-GR also confers cross-resistance to another EGFR-TKI. The mean IC50 values of four EGFR-TKI for $72 \mathrm{~h}$ growth inhibition assay in HCC827 and HCC827-GR cells. Data are representative of three independent experiments. (C) PHLPP Protein expression in HCC827 and HCC827-GR cell lines were detected by western blotting. (D) HCC827 and HCC827-GR cell lines were treated with increase concentration gefitinib for $24 \mathrm{~h}$. The cells were lysed and indicated protein were detected by western blotting. (E) HCC827 and HCC827-GR cell lines were treated with indicated EGFR-TKI for $24 \mathrm{~h}$. The cells were lysed and indicated protein were detected by western blotting.

TKI-induced apoptosis was decreased similar to gefitinib (Figure 3E). To explore the mechanism of gefitinib resistance induced by PHLPP loss, we next examined blocked phosphorylation level of EGFR, AKT, and ERK1/2 induced by gefitinib in both PHLPP knockdown and control HCC827 cells. We found that EGFR phosphorylation was completely abolished in both control and PHLPP-knockdown cells, but AKT and ERK1/2 phosphorylation substantially retained in the presence of gefitinib in PHLPP knockdown cells (Figure 3F). Taken together, our finding revealed that PHLPP downregulation attenuated the effect of EGFR-TKI, decreased the dephosphorylation levels of both AKT and MAPK, reactivated AKT and MAPK pathway in vitro, therefore, PHLPP downregulation decreased cell sensitivity to EGFR-TKI.

\section{Overexpression of PHLPP Increase Sensitivity to EGFR-TKI}

We next evaluated whether overexpression of PHLPP would restore sensitivity to EGFR-TKIs in the gefitinib resistance HCC827-GR cells. We established stable PHLPP overexpression cell line by using retrovirus-based transduction in HCC827-GR cells (Figure 4A).
PHLPP was increased by about 77 -fold after transduction. Overexpression of PHLPP was able to restore HCC827-GR sensitivity to gefitinib with a 17-fold decrease in the gefitinib IC50 compared to HCC827-control cells (Figure 4B). Similar results were obtained in a gefitinib intrinsic resistance cell line H1975 (Figure 4C), the IC50 value of gefitinib decreased by 8 -fold in PHLPP overexpression cells (Figure 4D). In addition, overexpression of PHLPP in H1975 significantly increased other EGFR receptor kinase inhibitors, afatinib and osimertinib-induced apoptosis (Figure 4E). Although EGFR phosphorylation was not inhibited by gefitinib, both AKT and ERK1/2 phosphorylation were effectively inhibited in PHLPP overexpression of H1975 cells (Figure 4F). Collectively, these findings strongly revealed that the ability of PHLPP to forestall and restore the onset of resistance to EGFR inhibitor via inhibiting EGFR downstream activation in both PI3K-AKT and MAPK-ERK signaling pathways.

\section{PHLPP Inhibition Advanced the Onset of Resistance to Gefitinib In Vivo}

To determine whether PHLPP affect cell sensitivity to gefitinib in vivo, we conducted tumor xenograft model using subcutaneously 
A

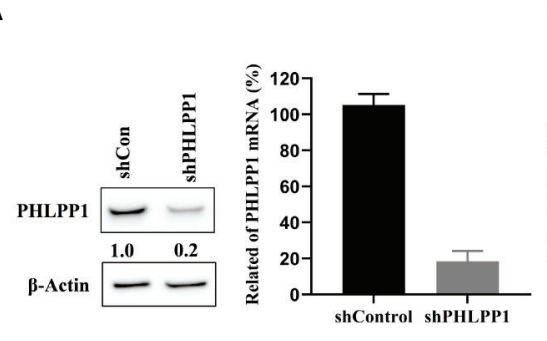$$
\text { B }
$$

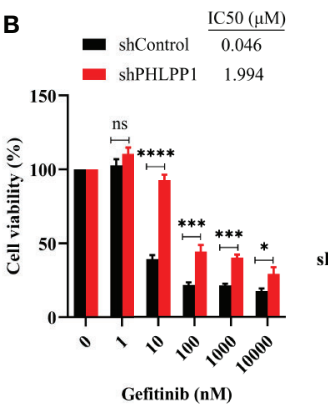

C

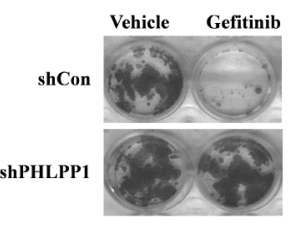

D

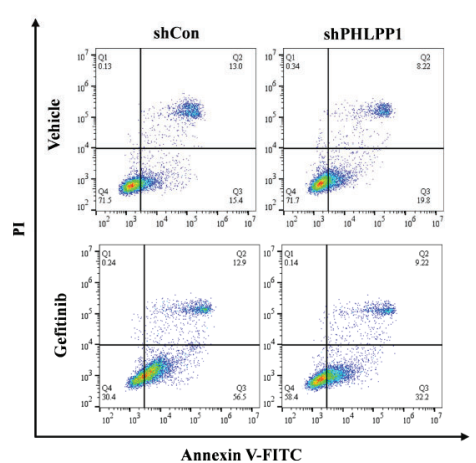

E

$\mathbf{F}$

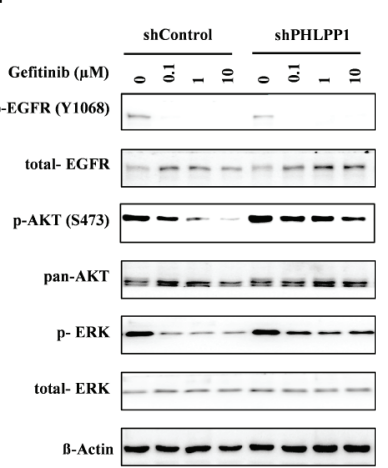

FIGURE 3 | Knockdown .of PHLPP decrease sensitive to EGFR-TKI in EGFR sensitive cell line. (A) Knockdown efficiency of PHLPP in HCC827 cell line as determine by both at mRNA level and protein level using quantitative RT-PCR and immunoblotting, respectively (B) IC50 values ( $\mu$ M) were based on data obtained from growth inhibition assay. (C) Longer-term colony formation assay using to determine downregulation PHLPP expression enhances cell survival (D) Detection of apoptosis with annexin V/flow cytometry. (E) HCC827-shControl and HCC827-shPHLPP cell lines were exposed to indicated EGFR-TKIs for $24 \mathrm{~h}$ then harvested for detection of apoptosis with annexin V/flow cytometry. The apoptosis of \% are mean \pm SE of triplicate assay. (F) HCC827-shControl and HCC827-shPHLPP cell lines were treated with increase concentration gefitinib for $24 \mathrm{~h}$. The cells were lysed and indicated protein were detected by western blotting.

tumor planting and following a 21-day gefitinib treatment (150 $\mathrm{mg} / \mathrm{kg}$, daily). The tumor growth rates were similar in both PHLPP knockdown and control HCC827 cells derived tumors (Figures 5A, B). Over 21-day gefitinib treatment, we found three of six mice in the control group but only one of six mice in PHLPP knockdown group had complete tumor regression (as detected by palpation), the mean tumor volumes in PHLPP knockdown group were higher than in control group $\left(4.6 \mathrm{~mm}^{3}\right.$ vs. $31.9 \mathrm{~mm}^{3}$, Figure 5C). Moreover, after the stop of gefitinib treatment, we observed no tumor growth up to 40 days in control group (Figure 5A) but tumor grow again within 1 month in PHLPP knockdown group (Figure 5B). Furthermore, we analyzed a microarray expression profiling of previously study that have been published by Zhang et al. (21), in which 17 xenograft tumors treated with erlotinib as well as two vehicletreated control tumors have been conducted. We found that there were 6 of the 17 (35\%) tumors with downregulation in HCC827 erlotinib-resistant tumor (Figure 5D). On the other side, PHLPP upregulation was not observed in those erlotinibresistant xenograft mice (21).

Taken together, these results further validation of reduced PHLPP expression alter tumor response to treatment and predictive onset of tumor recurrence in vivo.

\section{PHLPP Loss Is Associated With Resistance to EGFR Inhibitor and Poor Outcome in EGFR Mutant Patients}

To assess whether PHLPP expression associate with resistance to EGFR-TKI in clinical, we detected PHLPP expression levels in tumor samples using IHC technology, these pre- and postdevelopment samples were from patients who carry EGFR common mutations. Matched number of normal tissues as control. In 23 pretreatment and post-relapse tumor sections, we detected 10 of 23 (43.5\%) patients with T790M mutation (Figure 6A), 18 of 23 (78.3\%) patients showed decreased PHLPP expression at the time of the radiographical progression compared to pre-treatment (Figure 6B), none of patients with increased PHLPP expression. Of these 18 decreased PHLPP patients, $8(44.4 \%)$ patients with a T790M second mutation, which was similar as observed from those patients without a T790M second mutation. None of these were included in our previously published cohort of EGFR-TKI treatment patients (16).

In addition, to evaluate whether PHLPP is involved in the primary resistance in patients as well, we tested the PHLPP expression level taken at diagnosis using IHC. Using the median as cutoff, we found that PFS was drastically shorter in low 
A
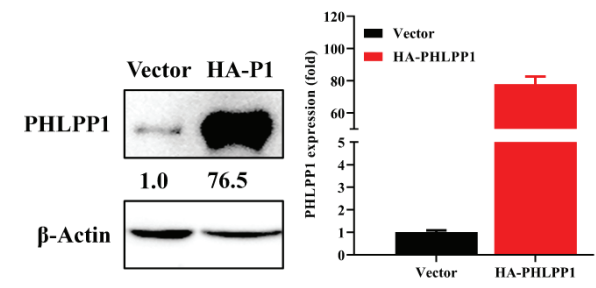

C
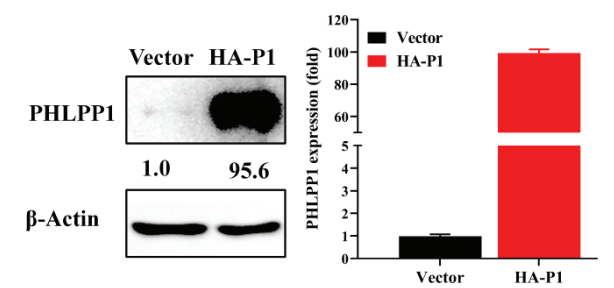

E

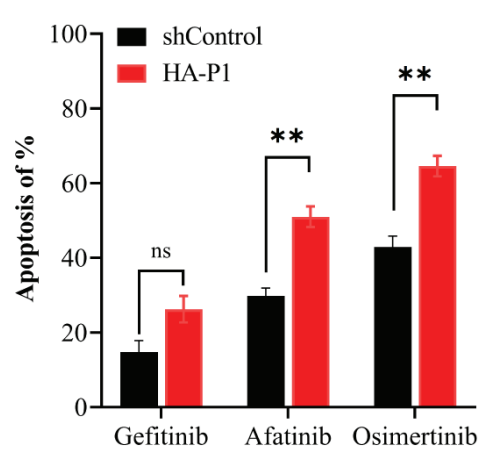

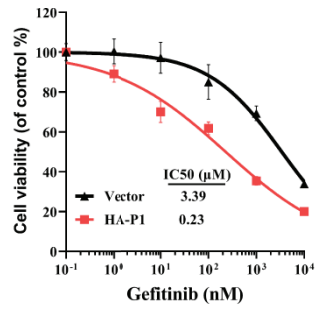

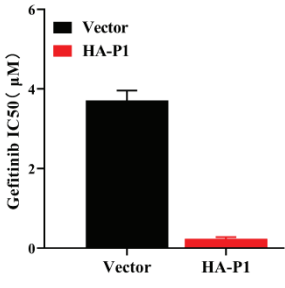

D
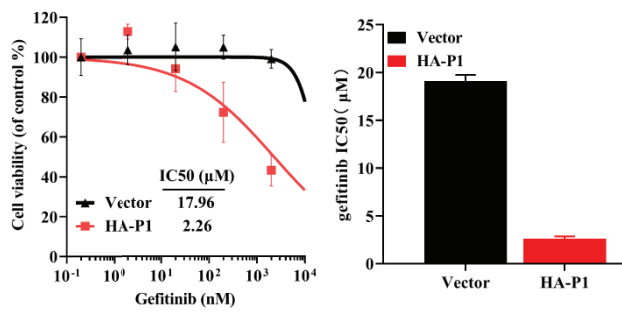

$\mathbf{F}$

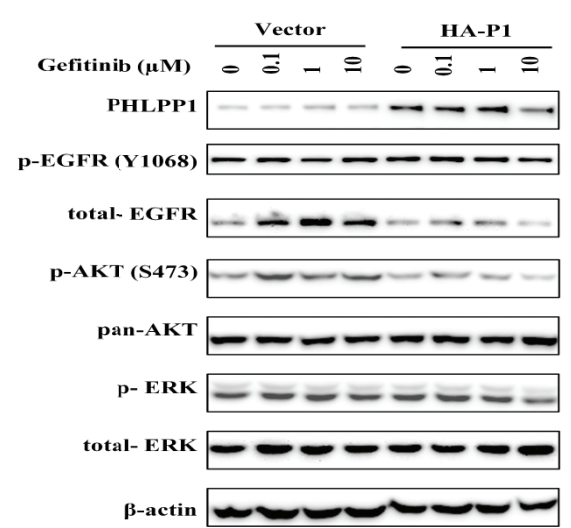

FIGURE 4 | Overexpression of PHLPP increase sensitivity to EGFR-TKI in gefitinib resistance cell line. (A) Overexpression efficiency of PHLPP in HCC82-GR cell line as determine by both at mRNA level and protein level using quantitative RT-PCR and immunoblotting, respectively (B) Cell-growth inhibition assay. All experiments were repeated at least three times. (C) Overexpression efficiency of PHLPP in H1975 as determine at protein level using immunoblotting (left) and fold changes from triplicate experiments (right) (D) Cell-growth inhibition assay. The gefitinib IC50 value are mean \pm SE of triplicate assay (right). (E) HCC827-GR cell were exposed to indicated EGFR-TKIs for $24 \mathrm{~h}$ then harvested for detection of apoptosis with annexin V/flow cytometry. The apoptosis of $\%$ are mean \pm SE of triplicate assay statistical testing was performed by two-sided t-test; ${ }^{* *} \mathrm{P}<0.005$, (F) H1975-Vector and H1975-HA-PHLPP cell lines were treated with increase concentration gefitinib for $24 \mathrm{~h}$. The cells were lysed and indicated protein were detected by Western blotting.

PHLPP expression group compared to high expression group (median PFS of 5.0 months vs 20.0 months, $\mathrm{p}=0.0001$, Figure 6C). Taken together, these clinical data suggest that PHLPP expression level significantly decreased after EGFRTKI therapy in clinical, it also can be used as a biomarker to predict the patient response to EGFR-TKI.

\section{DISCUSSION}

EGFR-TKI has demonstrated remarkable clinical activity in EGFR mutation NSCLC and has transformed the treatment paradigm for this highly prevalent disease in Asian populations. Despite this impact, intrinsic and acquired resistance to EGFR inhibitors remains a major challenge in the clinic. In the present study, we have identified loss of the PHLPP tumor suppressors as mechanisms of resistance to EGFR-TKI in NSCLC. Furthermore, PHLPP levels may influence the initial response to TKIs, as low as PHLPP in pre-treatment specimens is strongly associated with reduced PFS for patients with EGFRmutant lung adenocarcinomas treated with EGFR TKI.

A major clinical challenge in targeting EGFR-mutation NSCLC is resistance to EGFR blockade by multiple molecular mechanisms. The most frequents cause of EGFR-TKI resistance is the secondary mutations in EGFR, such as T790M attributed to the first- and secondary-generation EGFR-TKIs (17), C797S mutation confers resistance to irreversible third-generation EGFR-TKI (22). EGFR-independent mechanisms include 
A

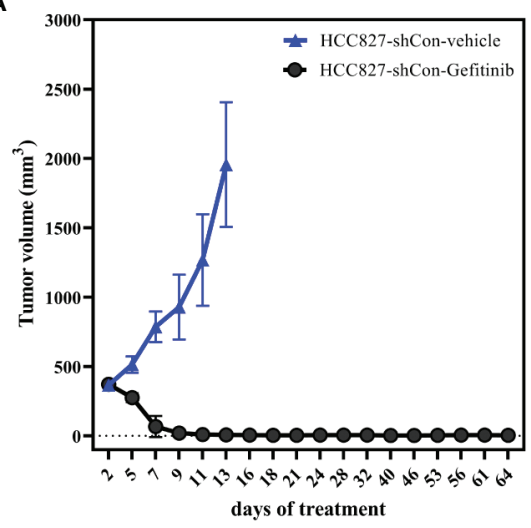

C

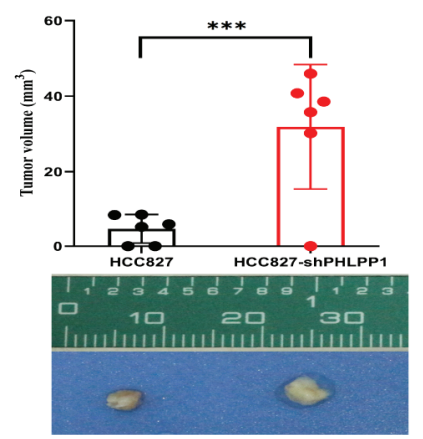

B

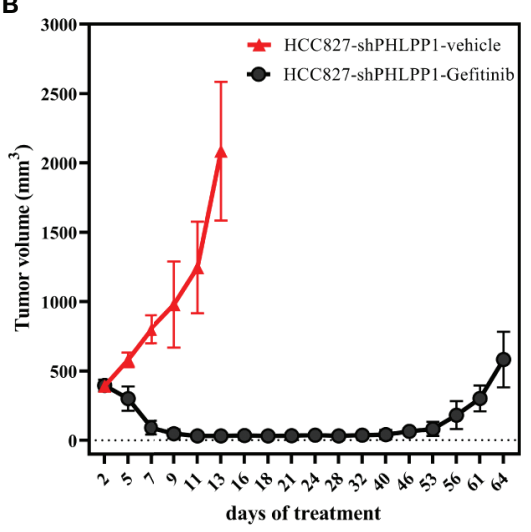

D

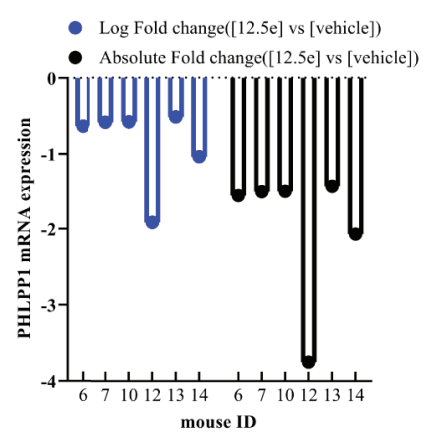

FIGURE 5 | Effect of PHLPP suppression on EGFR-TKI sensitivity in vivo. (A, B). Xenografts in nu/nu mice were generated from either HCC827-shControl (A) or HCC827-shPHLPP cells (B). Gefitinib (150mg/kg) was administered by oral gavage, and tumors volume were measured every other day. Mean tumor volumes were shown. (C) The mean residue tumor volume after 21-day course gefitinib treatment statistical testing was performed by two-sided t-test; ${ }^{\star \star \star} P<0.0005$. (D) The log fold and absolute fold change of PHLPP mRNA from a previously study have been published from Zhang et al.

bypass pathway or downstream activation by amplification of MET $(8,23)$, CRKL (24), HER2 (25), SMO (26), ERK (27) and KRAS (28)et al. Other altered molecular mechanisms involved in EGFR blockade resistance are transformation to small-cell lung cancer, epithelial-mesenchymal transition (10, 29-31), and the activation of immune system and other immunosuppressive factors, such as PD-L1 (32). In addition, a recently study reported stress hormone activation of $\beta 2$-AR promotes EGFR TKI resistance in cell lines and in mouse models of EGFR mutant NSCLC and that this can be blocked by anti-IL- 6 antibodies or $\beta$-blockers (33).

PHLPP downregulation occurs in several type cancers and is associated with poor clinical outcome and treatment resistance. In the present study, we found that PHLPP expression level positively correlated with EGFR-TKI sensitivity in EGFR mutation lung cancer. Among three EGFR mutation cell lines, we found that the expression of PHLPP in HCC827 cell line was the highest, H1975 cell line was the lowest, whereas gefitinib IC50 in H1975 cell line was highest and HCC827 cell line was lowest, respectively. Overexpression of PHLPP protein improves the efficacy of PI3K inhibitor (34)and rapamycin in colon cancer cells (35). Another study demonstrated that the level of PHLPP expression regulates drug sensitivity in colon cancer cells and that downregulation of PHLPP contributes to hypoxia-induced chemoresistance (15). More recently, it has been shown that PHLPP expression decreased in proteasome inhibitor bortezomib-resistance cells and bone marrow sample of patients with multiple myeloma, and overexpression of PHLPP partially increased bortezomib sensitivity in multiple myeloma cell lines (36). Here, we showed that overexpression of PHLPP in EGFR-TKIs resistance cell lines increased apoptosis induced by EGFR inhibitors. Downregulation of PI3K-AKT signaling is necessary for EGFR-TKI to effectively induce apoptosis (16). PHLPP is discovered for its tumor suppressor function in dampening both PI3K-AKT survival and ERK proliferative pathways $(12,13)$. It has been shown that AKT or/and ERK reactivation via varied mechanisms played important role in EGFR-TKI resistance (37). However, the mechanisms of reactivation of PI3K-AKT and MAPK-ERK pathway are unclear. Consistent with previous studies, we found that PHLPP is downregulated in gefitinib resistance cell lines accompanied by reactivation of AKT and ERK. Overexpression of PHLPP sensitize EGFR-TKI resistant cell line to EGFR inhibitors. Conversely, downregulation of PHLPP in EGFR-TKI sensitive cell lines decrease EGFR inhibitors induced apoptosis. 
A

\begin{tabular}{|c|c|c|c|c|c|c|c|}
\hline \multicolumn{1}{|c|}{ patient \# } & Sex & age & pre-mutation & post-mutation & pre-score & post-score & EGFR-TKI \\
\hline I & F & 63 & L858R & L858R & - & - & Gefitinib \\
\hline 2 & F & 68 & L858R & L858R & ++ & + & Gefitinib \\
\hline 3 & F & 58 & L858R & L858R & +++ & + & Erlotinib \\
\hline 4 & F & 70 & L858R & L858R/T790M & +++ & ++ & Gefitinib \\
\hline 5 & F & 59 & $19-d e l$ & $19-$ del/T790M & + & + & Gefitinib \\
\hline 6 & F & 60 & L858R & L858R & ++ & - & Gefitinib \\
\hline 7 & M & 60 & L858R & L858R/T790M & +++ & ++ & Gefitinib \\
\hline 8 & F & 56 & $19-d e l$ & $19-d e l$ & ++ & + & Gefitinib \\
\hline 9 & F & 70 & L858R & L858R/T790M & +++ & ++ & Icotinib \\
\hline 10 & M & 77 & L858R & L858R & ++ & + & Gefitinib \\
\hline 11 & F & 72 & $19-d e l$ & L858R & +++ & ++ & Gefitinib \\
\hline 12 & F & 62 & L858R & L858R/T790M & ++ & - & Icotinib \\
\hline 13 & M & 69 & $19-d e l$ & $19-$ del & + & - & Gefitinib \\
\hline 14 & M & 77 & L858R & L858R & +++ & ++ & Gefitinib \\
\hline 15 & M & 70 & L858R & L858R & ++ & + & Gefitinib \\
\hline 16 & M & 67 & L858R & L858R/T790M & ++ & - & Gefitinib \\
\hline 17 & F & 74 & L858R & L858R & ++ & ++ & Gefitinib \\
\hline 18 & M & 79 & L858R & L858R & - & - & Icotinib \\
\hline 19 & F & 51 & L858R & L858R/T790M & - & - & Gefitinib \\
\hline 20 & F & 59 & $19-d e l$ & L858R/T790M & + & - & Gefitinib \\
\hline 21 & M & 62 & $19-d e l$ & L858R/T790M & ++ & + & Gefitinib \\
\hline 22 & F & 52 & L858R & L858R & - & - & Gefitinib \\
\hline 23 & F & 74 & L858R & L858R/T790M & + & - & Icotinib \\
\hline & & & & & & & \\
\hline
\end{tabular}

B

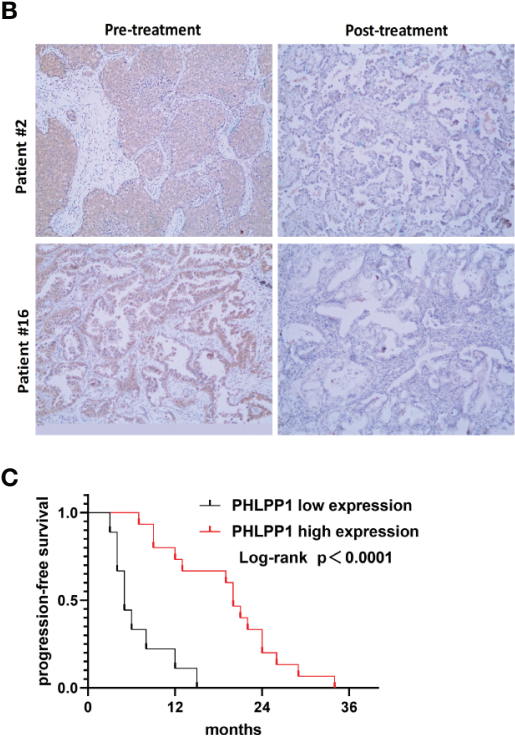

FIGURE 6 | Reduced PHLPP expression contributed to gefitinib resistance in clinical patients' tissue. (A) Clinical characteristics of 23 paired samples from patients with EGFR mutation lung cancer before and after the resistance to EGFR inhibitors. (B) Representative IHC Images of human EGFR mutation NSCLC tumor tissues with different PHLPP alteration stained with PHLPP antibodies. (C) Progression-free survival of patients receiving first line EGFR-TKI with high or low PHLPP expression level (as assessed by immunohistochemical analysis). Statistical significance was determined by Log-rank test.

Epigenetic dysregulation is in partly attributed to cancer treatment resistance, epigenetic drugs combined with molecularly targeted therapy could have important roles in reversing acquired therapy resistance. It has been shown that combinations of HDAC inhibitor such as vorinostat, panobinostat, and EGFR-TKIs to overcome EGFR-TKI resistance is associated with the BIM polymorphism in EGFRmutant NSCLC $(38,39)$. Another study has shown that osimertinib in combination with BET inhibitors JQ1 enhance the anti-tumor response in HER2 aberrations NSCLC (40). A preclinical research found that combination treatment with the EHMT2 inhibitor and erlotinib resulted in enhanced antitumor effects via reverse the silencing of PTEN and activation of the AKT pathway in EGFR-TKI-resistant cells in a preclinical EGFRTKI-resistance model (41). One limitation of our study is that we did not explore epigenetic. How chronic exposure to EGFR-TKI induces PHLPP expression decrease remains unclear and potentially involves transcription factors or chromatin modifying enzymes that regulate EGFR-TKI response. Dong et al. showed blockade of binding the transcription factor Sp1 to the PHLPP promoter contributes to DNA methylationmediated suppression of PHLPP and promotes Akt activation in melanoma (42).

\section{CONCLUSIONS}

In conclusion, our data point to activation of PI3K-AKT and MAPK-ERK signaling pathway by PHLPP loss as a potentially common mechanism of resistance to EGFR-TKI, nominating
PHLPP as a potential biomarker for EGFR-TKI sensitivity predictors, and implying that maintaining PHLPP expression during EGFR-TKI therapy may be a valuable strategy for delaying or overcoming drug resistance.

\section{DATA AVAILABILITY STATEMENT}

The raw data supporting the conclusions of this article will be made available by the authors, without undue reservation.

\section{ETHICS STATEMENT}

The studies involving human participants were reviewed and approved by the Institutional Medical Ethics Review Board, Taizhou Hospital of Zhejiang Province. The patients/ participants provided their written informed consent to participate in this study. The animal study was reviewed and approved by Institutional Animal Care and Use Committee, Taizhou Hospital of Zhejiang Province.

\section{AUTHOR CONTRIBUTIONS}

WW designed and performed most experiment, analyzed data, and wrote the manuscript, $\mathrm{XX}, \mathrm{KC}, \mathrm{MC}$, and $\mathrm{YM}$ performed some experiments in vitro assay and in vivo assay. DL performed experiment on clinical samples. HY designed and 
provided leadership for the project and wrote the manuscript. All authors contributed to the article and approved the submitted version.

\section{FUNDING}

This work was supported by Medical Science and Technology Project of Zhejiang Province, China (no. 2018KY887) and National Natural Science Foundation of China (no. 81874221).

\section{REFERENCES}

1. Siegel RL, Miller KD, Jemal A. Cancer Statistics, 2020. CA Cancer J Clin (2020) 70(1):7-30. doi: 10.3322/caac.21590

2. Chen J, Yang H, Teo ASM, Amer LB, Sherbaf FG, Tan CQ, et al. Genomic Landscape of Lung Adenocarcinoma in East Asians. Nat Genet (2020) 52 (2):177-86. doi: 10.1038/s41588-019-0569-6

3. Ramalingam SS, Vansteenkiste J, Planchard D, Cho BC, Gray JE, Ohe Y, et al. Overall Survival With Osimertinib in Untreated, Egfr-Mutated Advanced Nsclc. N Engl J Med (2020) 382(1):41-50. doi: 10.1056/NEJMoa1913662

4. Park K, Tan EH, O’Byrne K, Zhang L, Boyer M, Mok T, et al. Afatinib Versus Gefitinib as First-Line Treatment of Patients With EGFR Mutation-Positive Non-Small-Cell Lung Cancer (LUX-Lung 7): A Phase 2B, Open-Label, Randomised Controlled Trial. Lancet Oncol (2016) 17(5):577-89. doi: 10.1016/S1470-2045(16)30033-X

5. Mok TS, Wu YL, Thongprasert S, Yang CH, Chu DT, Saijo N, et al. Gefitinib or Carboplatin-Paclitaxel in Pulmonary Adenocarcinoma. $N$ Engl J Med (2009) 361(10):947-57. doi: 10.1056/NEJMoa0810699

6. Zhou C, Wu YL, Chen G, Feng J, Liu XQ, Wang C, et al. Erlotinib Versus Chemotherapy As First-Line Treatment for Patients With Advanced EGFR Mutation-Positive Non-Small-Cell Lung Cancer (OPTIMAL, CTONG-0802): A Multicentre, Open-Label, Randomised, Phase 3 Study. Lancet Oncol (2011) 12(8):735-42. doi: 10.1016/S1470-2045(11)70184-X

7. Ercan D, Zejnullahu K, Yonesaka K, Xiao Y, Capelletti M, Rogers A, et al. Amplification of EGFR T790M Causes Resistance to an Irreversible EGFR Inhibitor. Oncogene (2010) 29(16):2346-56. doi: 10.1038/onc.2009.526

8. Engelman JA, Zejnullahu K, Mitsudomi T, Song Y, Hyland C, Park JO, et al. MET Amplification Leads to Gefitinib Resistance in Lung Cancer by Activating ERBB3 Signaling. Science (2007) 316(5827):1039-43. doi: $10.1126 /$ science. 1141478

9. Niederst MJ, Sequist LV, Poirier JT, Mermel CH, Lockerman EL, Garcia AR, et al. RB Loss in Resistant EGFR Mutant Lung Adenocarcinomas That Transform to Small-Cell Lung Cancer. Nat Commun (2015) 6:6377. doi: 10.1038/ncomms7377. (In 11.47).

10. Raoof S, Mulford IJ, Frisco-Cabanos H, Nangia V, Timonina D, Labrot E, et al. Targeting FGFR Overcomes EMT-Mediated Resistance in EGFR Mutant Non-Small Cell Lung Cancer. Oncogene (2019) 38(37):6399-413. doi: 10.1038/ s41388-019-0887-2

11. Weng CH, Chen LY, Lin YC, Shih JY, Lin YC, Tseng RY, et al. EpithelialMesenchymal Transition (EMT) Beyond EGFR Mutations Per Se is a Common Mechanism for Acquired Resistance to EGFR Tki. Oncogene (2019) 38(4):455-68. doi: 10.1038/s41388-018-0454-2

12. Gao T, Furnari F, Newton AC. PHLPP: A Phosphatase That Directly Dephosphorylates Akt, Promotes Apoptosis, and Suppresses Tumor Growth. Mol Cell (2005) 18(1):13-24. doi: 10.1016/j.molcel.2005.03.008. (In eng).

13. Li X, Stevens PD, Liu J, Yang H, Wang W, Wang C, et al. PHLPP is a Negative Regulator of RAF1, Which Reduces Colorectal Cancer Cell Motility and Prevents Tumor Progression in Mice. Gastroenterology (2014) 146(5):130112.e1-10. doi: 10.1053/j.gastro.2014.02.003. (In eng).

14. Mussnich P, Rosa R, Bianco R, Fusco A, D'Angelo D. MiR-199a-5p and miR375 Affect Colon Cancer Cell Sensitivity to Cetuximab by Targeting PHLPP. Expert Opin Ther Targets (2015) 19(8):1017-26. doi: 10.1517/ 14728222.2015.1057569. (In 5.139).
The funder had no role in the study design, the data collection and analysis, the interpretation of the data.

\section{ACKNOWLEDGMENTS}

This work was partly presented at the IASLC 20th World Conference on Lung Cancer, September 7-10, 2019, Barcelona, Spain. We thank Tianyan Gao from University of Kentucky for providing several plasmids.

15. Wen YA, Stevens PD, Gasser ML, Andrei R, Gao T. Downregulation of PHLPP Expression Contributes to Hypoxia-Induced Resistance to Chemotherapy in Colon Cancer Cells. Mol Cell Biol (2013) 33(22):4594605. doi: 10.1128/mcb.00695-13. (In 4.777).

16. Xie Y, Lv D, Wang W, Ye M, Chen X, Yang H. High PHLPP Expression Levels Predicts Longer Time of Acquired Resistance to EGFR Tyrosine Kinase Inhibitors in Patients With Lung Adenocarcinoma. Oncotarget (2017) 8 (35):59000-7. doi: 10.18632/oncotarget.19777

17. Engelman JA, Mukohara T, Zejnullahu K, Lifshits E, Borras AM, Gale CM, et al. Allelic Dilution Obscures Detection of a Biologically Significant Resistance Mutation in EGFR-Amplified Lung Cancer. J Clin Invest (2006) 116(10):2695-706. doi: 10.1172/JCI28656. (In 13.215).

18. Coldren CD, Helfrich BA, Witta SE, Sugita M, Lapadat R, Zeng C, et al. Baseline Gene Expression Predicts Sensitivity to Gefitinib in Non-Small Cell Lung Cancer Cell Lines. Mol Cancer Res (2006) 4(8):521-8. doi: 10.1158/15417786.MCR-06-0095

19. Sirotnak FM, Zakowski MF, Miller VA, Scher HI, Kris MG. Efficacy of Cytotoxic Agents Against Human Tumor Xenografts is Markedly Enhanced by Coadministration of ZD1839 (Iressa), an Inhibitor of EGFR Tyrosine Kinase. Clin Cancer Res (2000) 6(12):4885-92. (In eng).

20. Lv D, Yang H, Wang W, Xie Y, Hu W, Ye M, et al. High PHLPP Expression is Associated With Better Prognosis in Patients With Resected Lung Adenocarcinoma. BMC Cancer (2015) 15:687. doi: 10.1186/s12885-015-1711-1

21. Zhang Z, Lee JC, Lin L, Olivas V, Au V, LaFramboise T, et al. Activation of the AXL Kinase Causes Resistance to EGFR-Targeted Therapy in Lung Cancer. Nat Genet (2012) 44(8):852-60. doi: 10.1038/ng.2330

22. Thress KS, Paweletz CP, Felip E, Cho BC, Stetson D, Dougherty B, et al. Acquired EGFR C797S Mutation Mediates Resistance to AZD9291 in NonSmall Cell Lung Cancer Harboring EGFR T790m. Nat Med (2015) 21(6):5602. doi: 10.1038/nm.3854. (In eng).

23. Wang Q, Yang S, Wang K, Sun SY. MET Inhibitors for Targeted Therapy of EGFR TKI-Resistant Lung Cancer. J Hematol Oncol (2019) 12(1):63. doi: 10.1186/s13045-019-0759-9

24. Cheung HW, Du J, Boehm JS, He F, Weir BA, Wang X, et al. Amplification of CRKL Induces Transformation and Epidermal Growth Factor Receptor Inhibitor Resistance in Human Non-Small Cell Lung Cancers. Cancer Discovery (2011) 1(7):608-25. doi: 10.1158/2159-8290.CD-11-0046

25. Takezawa K, Pirazzoli V, Arcila ME, Nebhan CA, Song X, de Stanchina E, et al. HER2 Amplification: A Potential Mechanism of Acquired Resistance to EGFR Inhibition in EGFR-Mutant Lung Cancers That Lack the Second-Site EGFRT790M Mutation. Cancer Discovery (2012) 2(10):922-33. doi: 10.1158/ 2159-8290.CD-12-0108

26. Della Corte CM, Bellevicine C, Vicidomini G, Vitagliano D, Malapelle U, Accardo M, et al. Smo Gene Amplification and Activation of the Hedgehog Pathway as Novel Mechanisms of Resistance to Anti-Epidermal Growth Factor Receptor Drugs in Human Lung Cancer. Clin Cancer Res (2015) 21 (20):4686-97. doi: 10.1158/1078-0432.CCR-14-3319

27. Li Y, Zang H, Qian G, Owonikoko TK, Ramalingam SR, Sun SY. ERK Inhibition Effectively Overcomes Acquired Resistance of Epidermal Growth Factor Receptor-Mutant Non-Small Cell Lung Cancer Cells to Osimertinib. Cancer (2020) 126(6):1339-50. doi: 10.1002/cncr.32655

28. Nakatani K, Yamaoka T, Ohba M, Fujita KI, Arata S, Kusumoto S, et al. KRAS and EGFR Amplifications Mediate Resistance to Rociletinib and Osimertinib in 
Acquired Afatinib-Resistant NSCLC Harboring Exon 19 Deletion/T790M in EGFR. Mol Cancer Ther (2019) 18(1):112-26. doi: 10.1158/1535-7163.MCT-18-0591

29. Sequist LV, Waltman BA, Dias-Santagata D, Digumarthy S, Turke AB, Fidias $\mathrm{P}$, et al. Genotypic and Histological Evolution of Lung Cancers Acquiring Resistance to EGFR Inhibitors. Sci Transl Med (2011) 3(75):75ra26. doi: 10.1126/scitranslmed.3002003

30. Yochum ZA, Cades J, Wang H, Chatterjee S, Simons BW, O'Brien JP, et al. Targeting the EMT Transcription Factor TWIST1 Overcomes Resistance to EGFR Inhibitors in EGFR-Mutant Non-Small-Cell Lung Cancer. Oncogene (2019) 38(5):656-70. doi: 10.1038/s41388-018-0482-y

31. Taniguchi H, Yamada T, Wang R, Tanimura K, Adachi Y, Nishiyama A, et al. AXL Confers Intrinsic Resistance to Osimertinib and Advances the Emergence of Tolerant Cells. Nat Commun (2019) 10(1):259. doi: 10.1038/ s41467-018-08074-0

32. Peng S, Wang R, Zhang X, Ma Y, Zhong L, Li K, et al. Egfr-TKI Resistance Promotes Immune Escape in Lung Cancer Via Increased PD-L1 Expression. Mol Cancer (2019) 18(1):165. doi: 10.1186/s12943-019-1073-4

33. Nilsson MB, Sun H, Diao L, Tong P, Liu D, Li L, et al. Stress Hormones Promote EGFR Inhibitor Resistance in NSCLC: Implications for Combinations With Beta-Blockers. Sci Transl Med (2017) 9(415):eaao4307. doi: 10.1126/scitranslmed.aao4307

34. Liu J, Weiss HL, Rychahou P, Jackson LN, Evers BM, Gao T. Loss of PHLPP Expression in Colon Cancer: Role in Proliferation and Tumorigenesis. Oncogene (2009) 28(7):994-1004. doi: 10.1038/onc.2008.450. (In eng).

35. Liu J, Stevens PD, Gao T. mTOR-dependent Regulation of PHLPP Expression Controls the Rapamycin Sensitivity in Cancer Cells. J Biol Chem (2011) 286 (8):6510-20. doi: 10.1074/jbc.M110.183087

36. Liu X, Li C, Fu Y, Liu J. Phlpp Sensitizes Multiple Myeloma Cells to Bortezomib Through Regulating Lamp2. Onco Targets Ther (2020) 13:40111. doi: 10.2147/OTT.S237343

37. Shi P, Oh YT, Deng L, Zhang G, Qian G, Zhang S, et al. Overcoming Acquired Resistance to AZD9291, A Third-Generation EGFR Inhibitor, Through
Modulation of MEK/ERK-Dependent Bim and Mcl-1 Degradation. Clin Cancer Res (2017) 23(21):6567-79. doi: 10.1158/1078-0432.CCR-17-1574

38. Nakagawa T, Takeuchi S, Yamada T, Ebi H, Sano T, Nanjo S, et al. Egfr-TKI Resistance Due to BIM Polymorphism can be Circumvented in Combination With HDAC Inhibition. Cancer Res (2013) 73(8):2428-34. doi: 10.1158/00085472.CAN-12-3479

39. Zang H, Qian G, Zong D, Fan S, Owonikoko TK, Ramalingam SS, et al. Overcoming Acquired Resistance of Epidermal Growth Factor ReceptorMutant Non-Small Cell Lung Cancer Cells to Osimertinib by Combining Osimertinib With the Histone Deacetylase Inhibitor Panobinostat (LBH589). Cancer (2020) 126(9):2024-33. doi: 10.1002/cncr.32744

40. Liu S, Li S, Hai J, Wang X, Chen T, Quinn MM, et al. Targeting HER2 Aberrations in Non-Small Cell Lung Cancer With Osimertinib. Clin Cancer Res (2018) 24(11):2594-604. doi: 10.1158/1078-0432.CCR-17-1875

41. Wang L, Dong X, Ren Y, Luo J, Liu P, Su D, et al. Targeting EHMT2 Reverses EGFR-TKI Resistance in NSCLC by Epigenetically Regulating the PTEN/AKT Signaling Pathway. Cell Death Dis (2018) 9(2):129. doi: 10.1038/s41419-0170120-6

42. Dong L, Jin L, Tseng HY, Wang CY, Wilmott JS, Yosufi B, et al. Oncogenic Suppression of PHLPP in Human Melanoma. Oncogene (2014) 33(39):475666. doi: 10.1038/onc.2013.420. (In eng).

Conflict of Interest: The authors declare that the research was conducted in the absence of any commercial or financial relationships that could be construed as a potential conflict of interest.

Copyright (c) 2021 Wang, Xia, Chen, Chen, Meng, Lv and Yang. This is an open-access article distributed under the terms of the Creative Commons Attribution License (CC BY). The use, distribution or reproduction in other forums is permitted, provided the original author(s) and the copyright owner(s) are credited and that the original publication in this journal is cited, in accordance with accepted academic practice. No use, distribution or reproduction is permitted which does not comply with these terms. 